\title{
Les écarts Romanow : certains se referment, d'autres pas
}

$\mathrm{L}$ es premiers ministres du Canada ont annoncé à la barre du jour le 16 septembre, avec grand soulagement, qu'ils ont entendu le message des contribuables qui en ont assez des longues listes d'attente et veulent que les gouvernements consacrent davantage de l'argent de leurs impôts aux soins de santé.

Les dépenses publiques fédérales pour les soins de santé augmenteront de 18 milliards de dollars en six ans et une formule d'indexation portera la somme à 41,2 milliards de dollars d'ici 2014. C'est suffisant - plus que suffisant pour ajuster les contributions fédérales que la Commission Romanow sur les soins de santé avait jugées trop maigres (ce qu'on a qualifié «d'écart Romanow»)'. L'engagement monétaire est apprécié : la majorité des experts conviennent que le financement du système canadien de santé est insuffisant. Cette mesure motivera-t-elle toutefois la «transformation» nécessaire pour répondre aux recommandations Romanow - et éliminer les autres écarts Romanow?

La somme semble importante, mais en fait, il s'agit d'une augmentation annuelle modeste - environ $3.5 \%$ du total des dépenses publiques consacrées aux soins de santé. Les dépenses à ce chapitre croissent cependant de $6 \%$ à $8 \%$ par année, et on s'attend généralement à ce que ces taux soient constants ou augmentent, à mesure que le tsunami de la nouvelle technologie submergera les cabinets et les hôpitaux. Même si les nouveaux fonds peuvent servir à raccourcir certaines des listes d'attente, à rafistoler les soins à domicile et à régler les coûts catastrophiques des médicaments, il est peu probable que le médecin ou le patient moyen remarquera une grande différence.

Avant de formuler ses recommandations, M. Romanow avait examiné attentivement les preuves mettant à jour les difficultés du système public de santé. Il en a évalué les ressources, puis a dressé un diagnostic et prescrit un programme de réadaptation, dont l'argent ne constitue qu'un volet : il a aussi constaté des failles qu'une injection d'argent ne peut éliminer. Or, ces failles n'ont nullement été abordées, ou à peine, au cours de la conférence sur les soins de santé en septembre. Les premiers ministres connaissent bien les problèmes du système public de santé et savent combien il est difficile de les régler, mais comme l'a affirmé sans détour le premier ministre de l'Alberta, Ralph Klein, «le seul point de convergence de la conférence a été l'argent, et rien d'autre». Cet échange intergouvernemental de style télévérité (même s'il s'est en fin de compte terminé à l'abri des caméras) devait produire un résultat, et le plus facile était de s'entendre sur l'argent. Le premier ministre a tenté d'intégrer à l'entente un certain contrôle de l'utilisation des transferts fédéraux, y compris, ce qui est important, un rapport national mesurant le rendement du système de santé et portant notamment sur les périodes d'attente. L'entente a cependant négligé les autres réformes que proposait $M$. Romanow.

Selon lui, la réforme exige que des changements fondamentaux soient apportés au mode de prestation des soins de santé. Les autres écarts Romanow sont notamment les suivants : entre les attentes du public et la viabilité réelle, entre la complexité des soins médicaux modernes et l'infrastructure d'information nécessaire pour diminuer l'inefficience, entre l'envergure actuelle de la pratique et le travail en équipe intégré désormais nécessaire. Dans ce dernier cas, on a à peine effleuré la question de la réforme des soins primaires. La réforme est un terme fort poli. Or, en réalité, il s'agit de redéfinir les rôles et responsabilités des prestateurs de soins de santé, en particulier des infirmières et des médecins.

La réforme est un éléphant qui dort dans le salon pendant que nous continuons de le contourner prudemment. La mise en œuvre des volets difficiles des réformes Romanow revient aux gouvernements provinciaux, aux professions et aux institutions en place, et notamment aux hôpitaux. Si nous voulons que les systèmes de soins de santé provinciaux survivent, le mode de collaboration entre professionnels doit changer. Apporter ces changements et d'autres (par exemple l'intervention du secteur privé) exigera l'engagement du public dont les attentes doivent aussi changer. Les budgets des soins de santé ne peuvent continuer de gonfler aux taux actuels supérieurs à l'inflation. Le Canada a besoin de discussions publiques informées et ouvertes, de données sur le fonctionnement du système de soins de santé et non des anecdotes les plus récentes de patients désespérés qui attendent une intervention pour une cataracte ou un cancer. Les Canadiens devront aussi aborder la question de leurs autres responsabilités collectives envers les générations à venir (qui porteront le fardeau des hausses de coûts) en matière d'éducation, de logement, de soin des enfants et de protection de l'environnement, tous aussi nécessaires que les soins médicaux pour la santé de la nation. - $7 A M C$

\section{Référence}

1. Romanow RJ. Guidé par nos valeurs - L'avenir des soins de santé au Canada. Saskatoon : Commission sur l'avenir des soins de santé au Canada, 2002. Disponible : www.commissionsoinsdesante.ca (consulté le 20 septembre 2004). 